\title{
Developing an information leaflet on 22q11.2 Deletion Syndrome for parents to use with professionals during health care encounters
}

\begin{abstract}
Purpose. The purpose of this project was to develop a parent to professional information leaflet for parents to share with professionals caring for children with $22 \mathrm{q} 11.2$ Deletion Syndrome (22q11.2 DS).
\end{abstract}

Research Design and Methods. A mixed method design was adopted. Data were collected by focused group interview and questionnaire with parents of children with 22q11.2 DS and an information leaflet was developed.

Results. Parents (48\%) identified the General Practitioner (GP) as the key health professional for which information was required.

Practice Implications. Parents should be involved in development of information leaflets in order to minimise 'repeated storytelling' during professional encounters.

Search Terms. 22q11.2 deletion syndrome, parent, rare disorder, information leaflet, research. 


\section{Introduction}

In the delivery of health care to children with complex needs or rare disorders, parents have long been regarded as experts in the care of their children (Darbyshire, 1994). The diagnosis of any rare disorder with chronic, complex care needs can bring major upheaval to the lives of the whole family and the family's needs for information is reported (Patterson Kelly \& Porock, 2005, Wahl, Banerjee, Manikam, Parlyo, \& Lakhanpaul, 2011).

In addition to challenges in care giving and its impact on the family, the ongoing provision of the child's care, whatever the diagnosis, also involves the development of a long-standing relationship between the child, family and many health professionals (Nuutila \& Salantera, 2006). In this relationship parents are often more knowledgeable about their child's disease and care than many healthcare professionals. Over time, parents also become experts, not necessarily in the disorder itself, but, in the way it is expressed in their individual child. As experts, parents identify the uniqueness of the child's signs and symptoms and recognise the need for an individual approach to their child's management and care. Mothers especially become attuned to, and familiar with, their particular child's signs and symptoms and how they affect the child's general health and wellbeing (Baker-Gomez, 2004) which others may not be aware of. Nicholl and Begley (2012), for example have reported on mothers "Knowingness" in children with complex needs and "Unknowingness" in others and the impact of this on the child and family. 
Parents may also find themselves informing and educating health professionals about their child's signs and symptoms and their specific needs. In health care encounters 'Repeated storytelling' also causes parents' concerns when they have to reiterate their child's history and illness symptoms repeatedly during multiple visits to different professionals (Nicholl \& Begley, 2012). Kirk \& Glendinning (2002) further outline that rather than being supportive in nature, some relationships with professionals are more stressful than beneficial, especially if parental experience and knowledge is not acknowledged. Children with complex care needs, including those with rare disorders require high levels of care for physiological, psychological and social care needs which means they and their families encounter a wide range of health, social and education professionals (Carter, Cummings, \& Cooper, 2007). Additionally, the need for shared information increases in healthcare encounters with children with rare disorders and the time available to talk with parents in these situations is limited (Ygge \& Arnetz, 2004). Given this context, the aim of this project was to develop an information leaflet in conjunction with parents to aid them and their children during consultations. This type of information leaflet may also provide a template useful for families experiencing other rare disorders.

In this project the focus was on 22q11.2 deletion syndrome (22q11.2 DS), which is classified as a rare disorder (McGarvey \& Hart, 2008). 22q11.2 deletion syndrome follows an autosomal dominant pattern of inheritance. Multiple terms exist to describe 
the same syndrome, including; velo cardio facial syndrome, DiGeorge sequence, Sedlackova syndrome, Caylor syndrome, conotruncal anomalies face syndrome (Robin \& Shprintzen, 2005; Shprintzen, 2005). In 22q11.2 DS, a deletion from chromosome 22 exists at band q11.2 (Robin \& Shprintzen, 2005). According to Shprintzen (2008) the phenotype shows variable expression with no case of the syndrome reporting all the same clinical findings. Consequently, individuals presenting with 22q11.2 DS can have a widely varied clinical course even among affected members of the same family, and symptoms involve many parts of the body (Yazdani, McGhee, \& Stiehm, 2011). For example, development of 3rd and 4th pharyngeal pouches also known as "DiGeorge sequence" is present in many infants. Individuals can have absent thymus and parathyroid glands, and present with immunodeficiency and a wide variety of congenital heart defects such as ventricular or atrial septal defects and Tetralogy of Fallot (Yazdani, McGhee, \& Stiehm, 2011). Others may present with "velocardiofacial syndrome" such as cleft palate with or without cleft lip, hypernasal speech, congenital heart disease, short stature and developmental delay (Yazdani, McGhee, \& Stiehm, 2011). Distinguishable facial features can include; a long face, hood eyebrows and a tubular nose. Some children present with isolated congenital heart disease or cleft palate, while others may only have borderline normal intelligence, developmental delays or learning disabilities (De Smedt et al, 2007) and later in life are at an increased risk of developing mental illnesses such as schizophrenia, depression, anxiety, and bipolar disorder (Fabbro et al 2012). With so many phenotypes evident, management of the child with $22 \mathrm{q} 11.2$ DS is complex requiring a multi-disciplinary approach to care and numerous consultations with professionals. 
22q11.2 DS affects an estimated 1 in 4,000 people (McDonald-McGinn \& Sullivan, 2011). Although, the condition may actually be more common than this estimate, because some people with a 22q11.2 deletion have few signs and symptoms and are not diagnosed with the disorder.

\section{Aim and Objectives}

The overall aim of the project was to develop a parent led 22q11.2 DS information leaflet for the diverse group of professionals who are encountered by parents of children diagnosed with 22q11.2 DS. The objectives were to:

- Identify information that parents of children with 22q11.2 DS wish to include in an information leaflet for use during health care encounters.

- Design and develop an information leaflet for parents and their children to use with professionals during health care encounters.

\section{Purpose}

The study involved investigating parents' experiences of communicating with healthcare professionals in order to aid in the development of the parent led information leaflet. The project was also influenced by the National Strategy of Service User involvement in the Irish Health Service 2008-2013 (2008) which identifies "patient involvement in their own care" (p.15) as one of the seven key goals. These study participants were parents of 
children, as they are often the child advocates in healthcare consultations. These contextual factors and parental requests for an information leaflet specific to their situation formed the basis for the study. It was identified that no culturally appropriate and context specific information leaflets for families of children with 22 q11.2 DS was available in Ireland. The project team identified that an information leaflet was needed. In addition and given its highly variable expression, it is possible that no two children with 22q11.2 DS will present with exactly the same signs and symptoms and therefore, the ability to individualise the leaflet was a key issue. Recognizing parental expertise in each individual child's case was also likely to elicit 'hidden' information required in health care encounters to build trust in parent to professional relationships.

\section{Ethics}

Ethical approval was obtained from the University of Dublin, Trinity College, Ireland.

\section{Methods}

A two phase, mixed method approach using a focused group interview and administration of a specially designed questionnaire was adopted for the research study.

\section{Sample}

Census sampling, sometimes known as total sampling, was used in this study. It was chosen for this project as it enabled the researchers to access all parents $(n=107)$ on the 
22q11.2 DS support group register in the Republic of Ireland. It was determined the total population was too small for any other sampling method.

\section{Recruitment}

Parents were recruited through the 22q11.2 DS support group in the Republic of Ireland. An advertisement was placed on the 22q11.2 DS support group website and an information pack was distributed to members of the support group by a designated gatekeeper. The pack contained a letter of invitation, an information leaflet and a consent form for participation in the focused group interview, with a stamped addressed return envelope. The gate keeper who was not involved in the study was selected by the research team as an administrative volunteer with the support group. She held the confidential lists of names and addresses of members of the 22q11.2 DS support group but did not have a position in the group. This was felt to be unbiased and to hold no influence over the parents who were being invited to participate in this project, therefore, maintaining the parents right to refuse or participate in the project.

\section{Phase I and II}

Phase I informed the development of a questionnaire that was used in Phase II. Fifteen parents identified by the gatekeeper were invited by letter/ e-mail to participate in a focused group interview. The gatekeeper then arranged their attendance at the venue. The first 8 respondents were invited to attend and gave both verbal and written informed 
consent. Phase II involved the postal administration of the specifically designed questionnaire. All remaining parents $(n=92)$ recorded on the support group database were advised of the study by a notice placed on the organisation's website. An information pack, containing an information leaflet, letter of invitation and the questionnaire was sent

to all parents. A stamped addressed envelope was included for return of the completed questionnaire. In order to maintain the anonymity of the participants', packs were distributed by the gatekeeper. Participants were advised that return of the completed questionnaire represented their informed consent.

\section{Data Collection}

\section{Phase I - Focused Group Interview}

In Phase I a focused group interview was undertaken with 8 parents; 2 fathers and 6 mothers, of children with 22q11.2 DS. Their children's ages ranged from 3 to 18 years. The focused group interview was conducted by a member of the research team experienced in interviewing, lasted 70 minutes and was digitally audio recorded. The interviewer was a Registered Children's Nurse which may have influenced the interaction in the group. However, this was also viewed as an advantage as it meant that she was familiar with the condition and the number of healthcare professionals parents encountered and also meant that parents did not have to provide explanations which helped the flow of the interview. In addition, the interview guide developed from the literature, particularly the work of Nicholl and Begley (2012) was used to provide direction in the focused group interview during which participants were asked to provide 
information on their children and their experiences with healthcare professionals. Parent's comments were also collected by a note taker to ensure that all views were recorded. This information was taken into consideration in the development of the questionnaire and subsequent information leaflet.

\section{Phase II - Parental Questionnaire}

The content of the questionnaire was developed from information gathered in the focused group interview, including data on the range of professionals they encountered, who their most frequent consultations were with and what difficulties they had during consultations. In particular parents reported on consultations with non-healthcare professionals. Therefore, the researchers included non-healthcare professionals in the questionnaire. Following development but before its use, the questionnaire was reviewed by the team and the gatekeeper and a small group of parents $(n=7)$ not involved in Phase I of the study. The questionnaire consisted of four sections that included closed and open ended questions. Section 1 elicited information about parents' experiences with healthcare professionals and about improving these encounters. Section 2 similarly elicited parents' experiences with non-healthcare professionals. Section 3 focussed on the information parents would find helpful in an information leaflet. Section 4 elicited demographic and biological information on both parents and the child. At the end of the questionnaire parents were also invited to add any further comments for consideration in the development of the leaflet. This was subsequently sent to 92 parents registered with 
the 22q11.2 DS support group and who had not already participated in the focused group interview yielding a response rate of $25 \%(n=23)$.

\section{Data analysis}

Quantitative data were analysed using the Statistical Package for the Social Sciences (SPSS V 18). All qualitative data were inputted to Microsoft Word and analysed using thematic content analysis. Using this method, the themes emerge from the data analysis and are not imposed by the researcher and it is a useful approach when analyzing data from focused groups interviews (Holloway \& Wheeler, 2009). This involved multiple stages including reading the data, making notes, organising data into groups and assigning meaning to it in themes and broader categories (Burnard,1991). This process was completed by two researchers independently to guard against bias and then cross checked for similarities and comparisons. Emerging themes were then discussed and agreed as common and central to the enquiry. Three key themes emerged from the data analysis; information on child as an individual, information on syndrome and experience of frustration. The themes and sub themes are presented in Table 1.

\section{Findings}

The findings provide focused evidence on parents' health care encounters when their child has a rare or chronic disorder. The relatively small sample size from the parent support group may render the findings of this project both context and participant specific, and may limit the applicability of the findings. However, despite this, the 
findings provided insight into parent's experiences of their healthcare encounters when their child has 22q11.2 DS and assisted in the development of the information leaflet. In order to present the findings with clarity, the key qualitative and quantitative data from both the focused group interview (Phase I) and questionnaire (Phase II) are reported together as results of both were combined to assist in the development of the information leaflet.

The focused group interview comprised of parents from the local Leinster region. Parents who completed the questionnaire in Phase II were from a varied national geographical profile. Both the interview participants and the questionnaire respondents were predominantly female. The age range of Phase I participant's children varied from 4 to 18 years. In Phase II, the age range varied from 4 to 22 years. In both phases, the age at the time of diagnosis of the children with 22q11.2 DS ranged from newborn to 13 years of age.

\section{Parents experiences of dealing with professionals}

In both phases all participants reported that they met with a wide range of health and nonhealth professionals about their child. Some consultations were reported positively. However, many difficulties were encountered also. In relation to the content of the information leaflet, three broad themes were identified in the qualitative data that informed its development; information on child as an individual, information on the syndrome and the experience of frustration. 


\section{Information on child as an individual}

Almost half $(47.8 \% / \mathrm{n}=11)$ of survey participants reported that they perceived that professionals had an apparent lack of awareness of their child's needs. Parents described having to repeatedly explain 22q11.2 DS to both health and non-health professionals. Just over half of parents reported $(56.5 \% / \mathrm{n}=13)$ that they perceived an apparent lack of awareness amongst professionals of the characteristics of 22q11.2 DS and how these affected the children and in particular their individual child. Parents indicated that it is particularly important that the child's individual characteristics are recognized by health professionals, specifically as the child's needs may change as they get older. Parents also wanted health professionals to be aware of the 'invisible' features of the disorder including the child's learning ability and the need to explain procedures in a way that the child can understand.

"I feel the child's needs change all the time as they get older. Their physical needs are almost always obvious but their social and life skills are forgotten about. This should be made clear in the leaflet" (Mother of 15 year old).

Parents suggested it would be helpful if health professionals did not assume that the child understands the syndrome and providing the child with time to talk and ask questions is needed during consultations. They indicated that the child's memory and recall may be poor so unless the child is provided with an opportunity to ask a question immediately he/she may forget the question. As a consequence interruptions from the child should be 
expected during conversations as they process the information slowly and should be encouraged to participate.

"Give the child time to talk and ask questions as child's memory is bad. He sometimes needs to butt in and ask the question straight away as he will forget" (Mother of 14 year old).

This lack of understanding may continue into adulthood so that, though the child may look grown up, this may not be so in terms of their cognitive ability. One parent indicated for example that her 18 year old did not understand what the deletion meant.

"Even though she is 18, she does not understand herself what the deletion is all about" (Mother of 18 year old).

Respondents suggested that communication about the child's individual needs could be improved if a summary of the child's unique characteristics was recorded in the front of his/her medical chart. The variation and individuality of the characteristics in each child should, parents suggested be similarly recorded in an information leaflet. 
"Need guide specific to the individual child (possible attachment to main leaflet), showing conditions that they have and those currently affecting the child" (Father of 4 year old).

\section{Information on the syndrome}

Half of the parents $(52.1 \% / \mathrm{n}=12)$ reported that they perceived that professionals they meet had an apparent lack of awareness of the syndrome and just over a third (34.7\% $/ n=8$ ) of parents reported that professionals also lack an awareness of the cause of 22q11.2 DS. They recommended that greater information on the disorder should be available and it would be helpful if health professionals were aware of both the variation in symptoms and also the range of investigations that the child is required to undergo. Parents would like professionals to know specifically the different names given to the disorder, what the syndrome is, how it usually manifests itself, the variation in symptoms, the age specific needs of a child with 22q11.2 DS, the types of conditions associated with the syndrome and that these children have changing complex needs.

(All healthcare professionals) "Should be aware of child's history i.e. summary at front of medical file" (Mother of 11 year old).

This was suggested so that parents could avoid the 'repeated storytelling'. Additionally, health professionals need to be aware that the child with 22q11.2 DS can have a constellation of potential difficulties. Parents reported that healthcare professionals need 
to be aware for example, that children have differing medical, educational, psychological and social needs and wanted to include specific medical information in the leaflet such as;

"Need to include information on - learning ability/ not reaching milestones/ cleft palate/ failure to thrive/ reflux/ hearing problem and infections/ heart problems/ low muscle tone/ prone to infections" (Mother of 14 year old).

"What are the possible symptoms with 22q deletion (emphasis on possible) mental health/attention span/ cardiac problems/ behaviour problems" (Mother of 4 year old).

\section{Experience of Frustration}

Parents identified factors that caused them frustration in encounters with health professionals including limited time for consultations, not being listened to and repeating information about the child. These factors were also considered when developing the information leaflet. Parents recognize that their child has complex needs and feel they should be viewed as not 'just overly worried' parents. Health professionals, they suggested should be aware that parents know their child best and consider, and act on, this information. 
"Mums and Dads have a gut feeling when it comes to their child and we know them best. When my child was diagnosed at 9, I was most of all angry because I had always known there was a reason e.g. failure to thrive until age 2" (Father of 14 year old).

Parents indicated that during consultations, they repeated information about their child, the syndrome and its signs and symptoms. This repetition was problematic and should be avoided.

"You should not be repeating information to people you meet all the time, but unfortunately you are" (Father of 4 year old).

\section{Development of the information leaflet}

The need for an information leaflet was supported by the parents who expressed the need specifically for an information leaflet to assist them in communicating their child's unique signs and symptoms. Parents outlined key information that should be provided for professionals and who they felt needed this information. The General Practitioner (GP) was identified by almost half of the parents $(48 \% / \mathrm{n}=11)$ as a key health professional needing information as it is the GP who deals with general issues and acts as the gateway to the many professionals involved in the child's management. 
The speech and language therapist was also considered by $14 \%$ of parents as requiring specific information. The pediatrician "who can make referrals" was also considered important by parents $(14 \% / \mathrm{n}=3)$ as the third professional for whom an information leaflet should be developed. Parents viewed this as important as there are a range of specialists involved in the care of the children with 22q11.2 DS. In addition to health professionals, parents also indicated the need for information for teachers;

"Teachers should understand what 22q11.2 DS is" (Mother of 5 year old).

Parents had concerns about their child's learning difficulties due to poor comprehension. However, this worry was balanced by an emphasis on the need for teachers to pace learning at the child's ability and the provision of educational supports for not only learning but also for continued education for the child. Thus, there was a need for teachers to be aware of the needs of the individual child.

\section{Information parents would like to convey in the information leaflet}

Having identified the specific information that parents viewed was needed, an information leaflet was developed that reflected the broad range of parental views. One parent suggested "keeping the leaflet as simple as possible" and another wanted "the inclusion of a list of symptoms or a list of all possible symptoms of 22q11.2 DS”. Parents 
requested that the leaflet should include information on allowances and entitlements for their child and "where to go for support". As an outcome, relevant web addresses were included in the leaflet to direct professionals to the information. To capture the perspectives of parents' some parental quotes were also incorporated into the leaflet.

\section{Outcome}

The outcome of the research project was that parents' views were ascertained and used to develop an information leaflet specific to children with $22 q 11.2$ DS. The information leaflet developed as an outcome of this small project will enable parents of individuals with 22q11.2 DS to improve communication with healthcare and non-healthcare professionals. The information leaflet will be used by parents where necessary in their healthcare encounters. The information leaflet is intended to alleviate some of the stress

parents feel when they have to repeat themselves to healthcare and non-healthcare professionals and explain 22q11.2 DS at various appointments they attend with their children. While the information leaflet is available electronically, parents requested a physical copy of the leaflet as they felt it was most useful to have it to hand to healthcare professional during consultations. Thus far, 300 copies of the information leaflet have been distributed to the support group for their use. The hard copy allows the parent to individualize the leaflet with their child's specific characteristics on an inset within each leaflet. It was particularly useful as each parent/child can individualize the information leaflet with their child's specific characteristics. The finished information leaflet follows an eight panel design and was compiled in adherence with the Irish National Adult Literacy Agency guidelines (2010). These guidelines are to ensure those with literacy 
difficulties are not disadvantaged by the leaflet's wording or design. The guidelines advise on vocabulary, logical flow, avoiding unnecessary detail and the use of clear design to make the leaflet attractive and easier to follow.

The leaflet also provides a little detail on the background of the development process. It also contains the following sub headings:

- What is 22q11.2 DS Deletion Syndrome?

- What parents of a child with $22 q 11.2$ DS would like health and non-health professionals to know?

- What parents said about making their interaction with healthcare professionals a more positive experience.

A one page leaflet was designed to be placed within the information leaflet for detailing the child's specific characteristics. As this is a loose leaflet it can be updated regularly and allows for changes to be documented as the child grows. Finally, sources of referenced material are included for professionals who wish to know more about the syndrome.

\section{Discussion}


The findings provide a valuable insight into the extent to which the parents of children with 22q11.2 DS are required to consult with a broad range of health professionals in relation to the health needs of their child. Due to the small sample size it is difficult to generalize the project findings. However, research has shown that parents of children with complex needs or who are technology dependent experience similar frustrations during healthcare encounters (Pain 1999, Plant \& Sanders, 2007, Nicholl, Doyle, Moran, \& Guilfoyle, 2013).

Given the highly variable expression of 22q11.2 DS, no two children will present with exactly the same signs and symptoms. Listening to parent's expertise in each individual child's case is therefore valuable in building trust within parent-professional relationships. This project identified that parents of children with 22q11.2 DS face particular challenges and often, explanations and repeating information about their child's condition become a motivational factor in seeking and acquiring the necessary leaflets that meet their child's needs. Parents who have a child diagnosed with 22q11.2 DS become experts in their child's needs but some parents reported healthcare encounters that are less than satisfactory. A more systematic coordinated approach to care of rare disorders as identified by Knight and Senior (2006) could reduce problems such as delayed diagnosis, poorly coordinated care and a lack of information. The part parents and families play in care delivery and organization of their child's care is identified as crucial in this study and good communication between families and healthcare professionals is essential for optimum healthcare delivery. 


\section{Limitations of the project}

The sample was drawn from an established database of persons who registered with the 22q11.2 DS Ireland support group, so the findings may not be transferable to other countries or cultures. As such, all findings need to be interpreted as being reflective of $25 \%(n=23)$ of the total sample $(n=92)$ that responded to the postal questionnaire and may be indicative of response bias. Participants' gender profile within this study was predominantly female which reflects who the primary caregiver tends to be. It is well documented that the primary caregiver of children with complex needs tends to be the mother (Nicholl \& Begley, 2012). Participants were not shown a copy of the completed information leaflet until after the first print run of copies which were then made available through the support group.

\section{Conclusion}

Information leaflets are traditionally developed by professionals for service users. The newly developed information leaflet from this study differs in that it is parent led and in turn this will benefit not only the professional but the child and family. The 22q11.2 DS information leaflet that emerged from this study will address needs as identified through the inclusion of parents in the decision making process of the leaflet development. It should enable parents of children diagnosed with 22q11.2 DS to communicate efficiently and effectively with professionals using a systematic approach that allows for individualism. Moreover, it will reduce the need for repeated explanations of the 
syndrome to every new professional of the many health professionals encountered by parents and their children diagnosed with 22q11.2 DS. This information leaflet is linked to the 22q11.2 DS Ireland Support group where it can be accessed by parents. The evaluation of its utility in practice will involve a follow up study.

\section{How might this information affect nursing practice}

Parents of children diagnosed with 22q11.2 DS deletion syndrome have multiple encounters with a range of professionals. In this study parents reported some difficulties at some of these encounters. These difficulties centre on "repeated storytelling" and gathering of information on the child by the professional which leads to frustration for parents. This study findings show that parents, particularly parents of children with rare disorders, are well positioned to assist in the development of information leaflets about their children. This may in turn reduce the stress of repeated storytelling. The 22q11.2 DS information leaflet that emerged from this study was developed by parents for professionals. We recommend that nurses embrace the knowledge of parents in working with professionals along the syndrome trajectory with their child. Parents should be encouraged and empowered to educate professionals in the same way professionals educate parents. In the current healthcare environment and with the increased recognition of parents/children as consumers of healthcare all efforts are necessary to make healthcare encounters as stress free and easy as possible for parents/children. Increasing parental/child involvement in healthcare encounters is a positive way to improve the parents/child's experience and to improve practice further realizing family-centered care which is the gold-standard in children's health care settings. Consumer participation in 
health research is also currently of great significance in consumer satisfaction. The parents involved in this study greatly supported developing partnerships in research projects. Parents have long been recognised as experts in their child's care and this leaflet development is a further step in acknowledging the uniqueness of parental expertise. 


\section{References}

Baker-Gomez, S. (2004). Strategies for Living with VCFS: The Chromosome 22q11.2 DS Deletion. USA: Desert Pearl Publishing.

Burnard, P. (1991). A method of analyzing interview scripts in qualitative research. Nurse Education Today, 11 (6), 461-466.

Carter, B., Cummings, J., \& Cooper, L. (2007). An exploration of best practice in multiagency working and the experiences of families of children with complex health needs. What works well and what needs to be done to improve practice for the future? Journal of Clinical Nursing, 16, 527- 539.

Darbyshire, P. (1994). Living with a sick child in hospital. London: Chapman and Hall.

Department of Health and Children (2008). National strategy for service user involvement in the Irish health service: 2008-2013. Dublin: The Stationary Office.

De Smedt, B., Devriendt, K., Fryns, J.P., Vogels, A., Gewellig, M., \& Swillen, A. (2007). Intellectual abilities in a large sample of children with Velo-Cardio-Facial Syndrome: an update. Journal of Intellectual Disability Research, 51(9), 666-670.

Fabbro, A., Rizzi, E., Schneider, M., Debbane, M., \& Eliez, S. (2012). Depression and anxiety disorders in children and adolescents with velo-cardio-facial syndrome (VCFS). European Child \& Adolescent Psychiatry, 21, 379-385.

Holloway, I., \& Wheeler, S. (2009). Qualitative Research in Nursing and Healthcare, 3rd Edition. UK: Wiley Blackwell. 
Kirk, S., \& Glendinning, C. (2002). Supporting 'expert' parents- professional support and families caring for a child with complex health care needs in the community. International Journal of Nursing Studies, 39(6), 625- 635.

Knight, A.W., \& Senior, T. P. (2006). The common problem of rare disease in general practice. Medical Journal of Australia, 185(2), 82-3.

McDonald-McGinn, D.M, \& Sullivan, K.E. (2011). Chromosome 22q11.2 deletion syndrome (DiGeorge syndrome/velocardiofacial syndrome). Medicine, 90(1), 1-18.

McGarvey, B., \& Hart, C. (2008). An investigation into the social support needs of families who experience rare disorders on the island of Ireland. Dublin: Rehab Care.

National Adult Literacy Agency (2010). Guidelines for Good Adult Literacy Work. Dublin: National Adult Literacy Agency.

Nicholl, H., \& Begley, C. (2012). Explicating Care giving by Mothers of Children with Complex Needs in Ireland: A Phenomenological Study. Journal of Pediatric Nursing, 27(6), $642-651$.

Nicholl H., Doyle C., Moran S., \& Guilfoyle, M.J. (2013). Identifying the types of technology that are used by children with intellectual disabilities and associated complex needs living at home in Ireland. British Journal of Learning Disabilities, 41, 229-236.

Nuutila, L., \& Salentera, S. (2006). Children with a long term illness: Parents' experiences of care. Journal of Pediatric Nursing, 21(2), 153-160. 
Patterson-Kelly, K., \& Porock, D. (2005). A survey of pediatric oncology nurses’ perceptions of parent educational needs. Journal of Pediatric Oncology Nursing, 22(1), $58-66$.

Pain, H. (1999). Coping with a child with disabilities from the parents'perspective: The function of information. Child: Care, Health and Development, 25, 299-312.

Plant, K. M., \& Sanders, M. R. (2007). Predictors of care-giver stress in families of preschool-aged children with developmental disabilities. Journal of Intellectual Disability Research, 51, 109-124.

Robin, N.H. \& Shprintzen, R.J. (2005). Defining the clinical spectrum of deletion 22q11.2. Disability Rehabilitation, 147, 90-96.

Shprintzen, R.J. (2005). Velo-cardio-facial syndrome. Progress in Pediatric Cardiology, $20,187-193$.

Shprintzen, R.J. (2008). Velo-Cardio-Facial Syndrome: 30 Years of Study. Developmental Disabilities Research Reviews, 14, 3-10.

Wahl, H., Banerjee, J., Manikam, L., Parlyo, C., \& Lakhanpaul, M. (2011). Health information needs of families attending the paediatric emergency department. Archives of Disease in Childhood, 96(4), 335-339.

Yazdani, S., McGhee, S.A., \& Stiehm, E.R. (2011). Chronic Complex Diseases of Childhood, A Practical Guide for Clinicians. Florida, USA: Brown Walker Press. 
Ygge, B.M., \& Arnetz, J.E. (2004). A study of parental involvement in pediatric hospital care: Implications for clinical practice. Journal of Pediatric Nursing, 19(3), 217-223. 